\title{
The Unanticipated Benefits of Behavioral Assessments and Interviews on Anxiety, Self- Esteem and Depression Among Women Engaging in Transactional Sex
}

\author{
Jayleen K. L. Gunn, Alexis M. Roth, Katherine E. Center, Sarah E. Wiehe
}

\begin{abstract}
Women engaging in transactional sex have disproportional mental health co-morbidity and face substantial barriers to accessing social services. We hypothesized that participation in a longitudinal research study, with no overt intervention, would lead to short-term mental health improvements. For 4-weeks, 24 women disclosed information about their lives via twice daily cell-phone diaries and weekly interviews. We used $t$ tests to compare self-esteem, anxiety, and depression at baseline and exit. Tests were repeated for hypothesized effect modifiers (e.g., substance abuse severity; age of sex work debut). For particularly vulnerable women (e.g., less educated, histories of abuse, younger initiation of sex work) participation in research conferred unanticipated mental health benefits. Positive interactions with researchers as well as discussing lived experiences may explain these effects. Additional studies are needed to confirm findings and identify mechanisms of change. This work contributes to the growing body of literature documenting that study participation improves mental health.
\end{abstract}

This is the author's manuscript of the article published in final edited form as: Gunn, J. K. L., Roth, A. M., Center, K. E., \& Wiehe, S. E. (2015). The Unanticipated Benefits of Behavioral Assessments and Interviews on Anxiety, Self-Esteem and Depression Among Women Engaging in Transactional Sex. Community Mental Health Journal, 1-6. http://doi.org/10.1007/s10597-015-9844-x 


\section{Introduction}

Transactional sex, selling or purchasing sex in exchange for money, drugs, food, shelter, or other items (Bobashev et al. 2009), has been linked to disproportionally high rates of mental health morbidity in women (Ward et al. 1999). Poor mental health has been associated with sexual and drug-seeking behaviors that increase susceptibility to sexually transmitted infection (STI) and human immunodeficiency virus (HIV) (Boyle et al. 1997; Centers for Disease Control \& Prevention [CDC] 2005; Hutton et al. 2004). For example, Seal et al. found that women with lower self-esteem tend to take more sexual risks (e.g., have sex without using condoms) with casual partners (Seal et al. 1997). Similarly, depressed persons are more likely to engage in binge drinking, using illegal drugs, having sex while using drugs, having sex with drug users, and engaging in transactional sex (Boyle et al. 1997; CDC 2005; Hutton et al. 2004).

Consequently, rates of STI/HIV are higher among individuals with mental health issues than the general population; 45\% of STI clinic patients have been diagnosed with a psychiatric disorder compared with only 25\% of the general population (Erbelding et al. 2004; Reeves et al. 2011). HIV incidence is four-fold higher among psychiatric out-patients than the general population (Beyeret al. 2007). Researchers have suggested that in order to reduce STI/HIV among vulnerable populations, including women engaging in transactional sex, we must improve their mental health (Kelly et al. 1993; Nyamathi and Flaskerud 1992).

The barriers to entering social and mental health services for women engaging in transactional sex are well documented. In general, about $15 \%$ of persons diagnosed with mental health disorders do not seek treatment because of financial barriers and, of those who initiate treatment, about 16\% drop out due to the financial burden (Mojtabai et al. 2011). Structural barriers (e.g., travel costs, office hours, and social stigma) also deter women from obtaining 
social service (Kurtz et al. 2005; Mojtabai et al. 2011; Roth et al. 2013). While programs to reduce these barriers are clearly needed, researchers have also begun to consider how therapeutic benefits, associated with research participation, can be harnessed to improve health outcomes (Clifford and Maisto 2000; Epstein et al. 2005; Lightfoot et al. 2007; Kurtz et al. 2013). Taking part in in-depth interviews and discussing personal experiences have been shown to improve one’s sense of purpose, self-awareness, and empowerment (Hutchinson et al. 1994). Sharing life events can improve mental health outcomes by helping individuals regain a sense of control and, consequently, better manage their emotional reactions to experiences (Lyubomirsky et al. 2006; Pennebaker and Graybeal, 2001). Furthermore, interviews have been shown to be healing for participants whether or not they are intended to be therapeutic (Campbell et al. 2004). Diarykeeping has been shown to increase self-reflection and result in heightened self-awareness (Kurtz et al. 2013; Lyubomirsky et al. 2006; Pennebaker and Graybeal, 2001; Stopka et al. 2004).

Women engaging in transactional sex in particular, may benefit from sharing their personal narrative because of the highly stigmatized nature of their work (Rappaport 1995). To date, no paper has described how participation in studies may improve mental health outcomes among these women. The objectives of this paper are two-fold, 1) to describe psychological benefits associated with participation in a daily diary and interview study and 2) identify personlevel characteristics that moderated effects.

\section{Methods}

Data for this study were taken from a previously described 4-week study of women engaging in transactional sex (Roth et al. 2014).

\section{Participants and Recruitment}


Eligible women were English-speaking and at least 18 years of age; reported exchanging sex for drugs, money, or items of daily living within the preceding 90 days; expressed a likelihood that they would engage in commercial sex work again in the next 30 days; and reported being able to safely store and charge a cellular phone. Women were recruited via targeted outreach, venue-based recruitment, and incentivized peer referral.

\section{Cell phone diaries and interviews}

Participants disclosed information about their lives twice a day, approximately every 12 h, via a digital diary completed on a study-provided cell phone (Roth et al. 2014). The diaries assessed mood; alcohol/drug craving and use; and partnered sexual behaviors—including condom use, partner characteristics, and locations of sexual events. When no sexual activity was reported, participants completed questions about other activities to minimize differences in the length of daily participation.

Participants also completed weekly face-to-face interviews with study personnel. The interviewers in this study were two female public health researchers with professional backgrounds in case management with marginalized populations (e.g., persons living with HIV, children with behavioral health problems). The semi-structured interviews focused on the context of the women's sexual lives using information from the daily diaries to facilitate conversation. During the interviews, participants described their most recent sexual activity, as well as the locations and circumstances surrounding each sexual event. Due to the individualized nature of these interviews, rapport developed quickly between the interviewer and participants. In many instances, women disclosed other aspects of their lives (e.g. rationale for entering into sex work, familial relationships, feelings toward recent arrest, etc.). Although interviews were designed to be structured, interviewers allowed for deviation from the structure, allowing participants the 
latitude to fully discuss personal life events. Over the course of the study, participants described their involvement in this study as cathartic. To test for potential effects associated with research participation, baseline mental health measures were repeated in the exit interview.

\section{Measures}

Participants completed demographic, behavioral, and mental health measures at the baseline and exit interviews via computer-assisted self-interviews. Researchers were present to address technical difficulties.

Covariate measures. Measures from the baseline survey included: demographics, overall health, social support, sexual health (sexual abuse during childhood and sexual anxiety), sex work history (age of first sex work—did women start sex work before or after 18 years—and frequency of sex work within 90 days), substance use (within 90 days), and criminal history. Sexual anxiety was scored on a four-point Likert scale (Fortenberry et al. 2005). As there is no cutoff provided by the scale a natural break in the data was used, with scores in the top half ( $\geq 9$ out of 16) considered high in this study. Social support—a scale assessing perceived support from a significant other, family, and friends—-was measured on a seven point Likert scale using the 12-item Multidimensional Scale of Perceived Social Support, $\alpha=0.88$ (Zimet et al.1988). As there is no cutoff provided by the scale, and there was no natural break in the data, social support scores in the bottom quartile ( $\leq 2.6$ out of 7$)$ were considered low for this study.

Outcome measures. These included self-esteem, depression and anxiety. These items were included in the baseline survey and 4-weeks later at exit.

Self-esteem. Self-esteem was assessed using the 10-item Rosenberg Self-Esteem (RSE) Scale, a widely used measure of global self-esteem assessing how a person feels about him or herself (Schmitt and Allik 2005). The RSE is scored on a four-point Likert scale and includes 
items such as "I feel I'm a person of worth, at least on an equal basis with others" (Rosenberg 1979). It has an alpha coefficient of 0.88 (Schmitt and Allik 2005).

Depression. Depression was assessed using the nine-item Patient Health Questionnaire (PHQ-9), which measures depression on a four-point Likert scale and includes items such as "Over the last two weeks, how often have you been bothered by little interest or pleasure in doing things?” (Kroenke and Spitzer 2002). The PHQ-9 has an alpha coefficient of 0.89 (Kroenke2003).

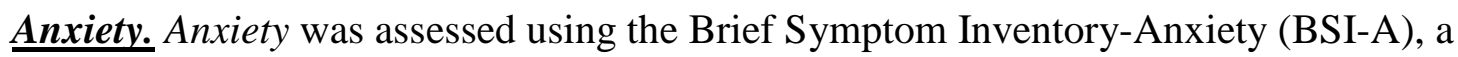
six-item scale rated on a five-point Likert scale (Derogatis and Melisaratos 1983). This scale includes items such as "How often do you feel nervousness or shakiness inside?" (Derogatis and Melisaratos 1983). The BSI-A has an alpha coefficient of 0.81. In this study, anxiety scores of 13 or higher (out of 24) were considered high due to a natural break in the data that clearly indicated a differences of high vs low scores in our sample.

\section{Analysis}

Data analysis was completed using Stata 12 (StataCorp, 2011). Paired t-tests were used to compare mental health scores (self-esteem, anxiety, and depression) from baseline to exit. Next, these tests were repeated for each stratum of the hypothesized effect modifiers. For example, when looking at the effect of social support on self-esteem, the sample was divided into two groups: those who had high social support at baseline and those who did not. Two t-tests were conducted to compare the self-esteem scores at the beginning and end of the study, one for women who had high social support and one for women who did not. All authors certify that there are no known conflicts of interest and that they are responsible for this study. This study 
was approved by the Indiana University Institutional Review Board and all participants provided written informed consent.

\section{Results}

\section{Sample characteristics}

Participants (N=24) were primarily middle-aged (median $=42.5)$, black $(75 \%)$, and nonLatina (87\%). In the 90 days preceding enrollment, 58\% of women engaged in transactional sex at least once a week. Substance use was prevalent; $25 \%$ of the sample consumed four or more alcoholic drinks per day; 25\% also used marijuana daily, and 42\% used cocaine weekly. All participants reported being arrested at least once; with over half reporting at least one arrest for sex work or drug charges (54\% each). Fifty-eight percent of participants reported experiencing childhood sexual abuse before age 13 and 42\% initiated sex work as minors (before age 18). Some FSW reported receiving little social support from family or friends (37\%). At baseline, the mean self-esteem score was 27 (out of 40), the mean depression score was 9 (out of 27) and 33\% of women in this study reported feeling high anxiety.

\section{Improvements in mental health over time}

Overall, self-esteem improved significantly; increasing 4.08 points from baseline to exit $(\mathrm{p}<.001)$. Mean scores for depression and anxiety decreased from baseline; however, these changes were not statistically significant overall. Nonetheless, import effect modification was observed for self-esteem, depression and anxiety.

\section{Stratified effect modifiers affecting improvement in mental health scores}

Personal history stratification. During this study, women who initiated sex work as minors reported decreased depressive scores (4.1 points, $\mathrm{p}=.05$ ). A history of sexual abuse in childhood did not moderate change in self-esteem, as increased self-esteem was seen in those 
who reported childhood sexual abuse (4.6 points, $\mathrm{p}=.00$ ) and those who did not (3.4 points, $\mathrm{p}=.05)$. Still, by the end of the study increases in self-esteem were found among women who initiated sex work as adults (5.5 points, $\mathrm{p}=.00)$ and those who had a 12th grade education (5.1 points, $\mathrm{p}=.00$ ) or below (4.8 points, $\mathrm{p}=.01$ ). Participating in this study did not significantly affect anxiety scores for women in any of these groups.

Substance use stratification. Over the course of the study, anxiety decreased in women who drank less than four alcoholic drinks a day (1.9 points, $\mathrm{p}=.03)$ and those who used marijuana daily (3.7 points, $\mathrm{p}=.05$ ). Increased self-esteem was found in women who drank less than four alcoholic drinks a day (4.7 points, $\mathrm{p}=.00$ ) and those who did not use cannabis daily (4.6 points, $\mathrm{p}=.00)$. Self-esteem also increased during this study for women whether they used cocaine weekly (4.9 points, $\mathrm{p}=.00$ ) or not (3.5 points, $\mathrm{p}=.02$ ). Participating in this study did not significantly affect depression scores for women in any of these groups.

Social support stratification. Self-esteem increased during this study for women who had high overall social support (3.8 points, $\mathrm{p}=.05$ ) and for those who did not (4.2 points, $\mathrm{p}=.00$ ). An increase in self-esteem was also found in women who received low social support from their significant other (3.8 points, $\mathrm{p}=.00$ ). Participating in this study did not significantly affect depression or anxiety scores for women in any of these groups.

\section{Discussion}

In this exploratory study we found that allowing women engaging in transactional sex to share their daily activities via daily diaries and weekly interviews improved mental health outcomes. Even with a very small sample, women experienced significant improvements in critical mental health measures_anxiety, depression, and self-esteem over a relatively short period of time. Stratifying the sample based on personal history, perceived social support, and 
substance use allowed us to assess which psychosocial characteristics were associated with changes in particular mental health scores.

We found a mean self-esteem at baseline to be 27 . This is lower than what we expected based on previous studies that report mean self-esteem of about 32 in adult populations (Schmitt and Allik 2005; Twenge and Campbell 2001). Increasing self-esteem 3-5 points brings the mean self-esteem scores in this sample close to that previously found in adults. This study was able to demonstrate a meaningful increase in self-esteem without a formal intervention. Also, the mean depression score in this sample was nearly twice that typically previously reported with the PHQ-9 (M=9 and M=5, respectively) (Kroenke et al. 2001). A 4 point decrease in depression for women who initiated sex work as minors brings the mean depression score in this group of women close to that found in other adult samples. Furthermore, anxiety is high in this sample, with 25-33\% of these women reporting high anxiety, while in the general public anxiety disorders occur in less than 10\% of women (McLean et al. 2011).

Although there was no overt mental health intervention in this study, there are multiple reasons to believe study methods elicited changes in mental health. Participating in interviews has been found to be healing for participants even when the interviews were not intended as therapeutic (Campbell et al. 2004). Sharing life events may help individuals regain a sense of control and better manage their emotional reactions to an experience (Kurtz et al. 2013; Lyubomirsky et al. 2006; Pennebaker and Graybeal, 2001; Stopka et al. 2004). Diary-keeping has been shown to increase self-reflection, resulting in heightened self-awareness (Kurtz et al. 2013; Lyubomirsky et al. 2006; Pennebaker and Graybeal, 2001; Stopka et al. 2004). Based on these results, research participation can be utilized to improve mental health outcomes and this 
type of intervention may be more successful targeting particular sub-populations of women who engage in transactional sex.

Previous research has already suggested that patients' psychosocial needs are one of the underlying determinants of health, including STI/HIV prevention (Chudakov et al. 2002; Sobel 1995). Women who are trading sex for drugs, money, or items of daily living are unlikely to have the means to pay for therapy. Moreover, women engaging in transactional sex confront structural (e.g., travel costs, office hours, and social stigma) and physical (e.g., drug use, mental stability, and fear) barriers to obtaining social services (Mojtabai et al. 2011; Roth et al. 2013). In addition, of those with mental health disorders who seek treatment, about $16 \%$ drop out due to cost (Mojtabai et al. 2011). Furthermore, there have been less social stigma attached to participating in research study than accessing more traditional therapeutic services; the interviews were conducted within the community in locations that were familiar to participants, researchers were accustomed to working with high-risk female populations and non-judgmental attitudes towards the women’s sexual lives. Given these women often experience negative interactions with clinical staff, it may be they felt more comfortable outside of a clinical setting (Roth et al. 2013).

Our findings should be considered within the limits of the design. The small sample size of this feasibility study $(\mathrm{N}=24)$ limits the power of this study to detect differences across groups. The relatively short study period limited the amount of interaction between participants and researchers, and our ability to assess whether changes in mental health were sustained over time. Therefore, it is unknown if the improvements in mental health seen here were lasting changes in the mental health of our participants or if it was simply a short term by-product of being under surveillance (Fernald et al. 2012; Gittelsohn et al. 1997). Future studies can address these 
limitations by enrolling more participants in studies of longer duration and following participants after the interactions have ended, in order to assess whether any observed changes in mental health were a by-product of being under surveillance. Finally, these participants completed the diaries and interviews concurrently; thus, there is no way in the present study to tease apart the effects of participants' diary-keeping and weekly interviews on the observed changes in mental health scores. While positive interactions with researchers, as well as discussing women's lived experiences, may explain these effects, more research is needed to understand which participants would benefit most from participating in this kind of technique.

\section{Conclusion}

Allowing women engaging in transactional sex to share their daily activities and personal narratives improved mental health outcomes. Even with a very small sample, FSWs experienced significant improvements in critical mental health measures—anxiety, depression, and selfesteem. Stratifying the sample based on personal history, perceived social support, and substance use illuminated the psychosocial characteristics associated with specific outcomes. These findings have important implications for others interested in engaging high-risk populations to improve mental health outcomes who are unlikely to seek out or continue with traditional therapeutic interventions. 


\section{References}

Beyer, J. L., Taylor, L., Gersing, K. R., \& Krishnan, K. R. R. (2007). Prevalence of HIV infection in a general psychiatric outpatient population. Psychosomatics, 48, 31-37.

Bobashev, G. V., Zule, W. A., Osilla, K. C., Kline, T. L., Wechsberg, W. M., (2009). Transactional sex among men and women in the south at high risk for HIV and other STIs. Journal of Urban Health, 86, 32-47.

Boyle, F. M., Dunne, M. P., Najman, J. M., Western, J. S., Turrell, G., Wood, C., \& Glennon, S. (1997). Psychological distress among female sex workers. Australian and New Zealand Journal of Public Health, 21, 643-646.

Campbell, R., Sefl, T., Wasco, S. M., \& Ahrens, C. E. (2004). Doing community research without a community: Creating safe space for rape survivors. American Journal of Community Psychology, 33, 253-261.

Centers for Disease Control \& Prevention (CDC). (2005). Mental health in the United States: Health risk behaviors and conditions among persons with depression--New Mexico, 2003. MMWR. Morbidity and Mortality Weekly Report, 54, 989-991.

Chudakov, B., Ilan, K., Belmaker, R. H., \& Cwikel, J. (2002). The motivation and mental health of sex workers. Journal of Sex and Marital Therapy, 28, 305-315.

Clifford, P. R. \& Maisto, S. A. (2000). Subject reactivity effects and alcohol treatment outcome research. Journal of Studies on Alcohol, 61, 787-793.

Derogatis, L. R. \& Melisaratos, N. (1983). The brief symptom inventory: An introductory report. Psychological Medicine, 13, 595-605.

Epstein, E. E., Drapkin, M. L., Yusko, D. A., Cook, S. M., McCrady, B. S., Jensen, N. K. (2005). Is alcohol assessment therapeutic? Pretreatment change in drinking among alcoholdependent women. Journal of Studies on Alcohol, 66, 369-378.

Erbelding, E. J., Hutton, H. E., Zenilman, J. M., Hunt, W. P., \& Lyketsos, C. G. (2004). The prevalence of psychiatric disorders in sexually transmitted disease clinic patients and their association with sexually transmitted disease risk. Sexually Transmitted Diseases, 31, 8-12.

Fernald, D. H., Coombs, L., DeAlleaume, L., West, D., \& Parnes, B. (2012). An assessment of the Hawthorne Effect in practice-based research. The Journal of the American Board of Family Medicine, 25, 83-86.

Fortenberry, J. D., Sayegh, M. A., \& Orr, D. P. (2005). The association of sexual self-concept with oral-genital sex among adolescent women. Journal of Adolescent Health, 36, 110.

Gittelsohn, J., Shankar, A. V., West, K. P., Ram, R. M., \& Gnywali, T. (1997). Estimating reactivity in direct observation studies of health behaviors. Human Organization, 56, 182-189. 
Hutchinson, S. A., Wilson, M. E., \& Wilson, H. S. (1994). Benefits of participating in research interviews. Image: The Journal of Nursing Scholarship, 26, 161-166.

Hutton, H. E., Lyketsos, C. G., Zenilman, J. M., Thompson, R. E., \& Erbelding, E. J. (2004). Depression and HIV risk behaviors among patients in a sexually transmitted disease clinic. American Journal of Psychiatry, 161, 912-914.

Kelly, J. A., Murphy, D. A., Bahr, G. R., Koob, J. J., Morgan, M. G., Kalichman, S. C., . . . St Lawrence, J. S. (1993). Factors associated with severity of depression and high-risk sexual behavior among persons diagnosed with human immunodeficiency virus (HIV) infection. Health Psychology, 12, 215-219.

Kroenke, K. \& Spitzer, R. L. (2002). The PHQ-9: A new depression diagnostic and severity measure. Psychiatric Annals, 32, 509-515.

Kroenke, K., Spitzer, R. L., \& Williams, J. B. (2001). The PHQ-9. Journal of General Internal Medicine, 16, 606-613.

Kroenke, K., Spitzer, R. L., \& Williams, J. B. (2003). The Patient Health Questionnaire-2: Validity of a two-item depression screener. Medical Care, 41, 1284-1292.

Kurtz, S. P., Surratt, H. L., Buttram, M. E., Levi-Minzi, M. A., \& Chen, M. (2013). Interview as intervention: The case of young adult multidrug users in the club scene. Journal of Substance Abuse Treatment, 44, 301-308.

Kurtz, S. P., Surratt, H. L., Kiley, M. C., \& Inciardi, J. A. (2005). Barriers to health and social services for street-based sex workers. Journal of Health Care for the Poor and Underserved, 16, 345-361.

Lightfoot, M., Rotheram-Borus, M. J., Comulada, S., Gundersen, G., \& Reddy, V. (2007). Selfmonitoring of behaviour as a risk reduction strategy for persons living with HIV. AIDS Care, 19, 757-763.

Lyubomirsky, S., Sousa, L., \& Dickerhoof, R. (2006). The costs and benefits of writing, talking, and thinking about life's triumphs and defeats. Journal of Personality and Social Psychology, 90, 692-708.

McLean, C. P., Asnaani, A., Litz, B. T., \& Hofmann, S. G. (2011). Gender differences in anxiety disorders: Prevalence, course of illness, comorbidity and burden of illness. Journal of Psychiatric Research, 45, 1027-1035.

Mojtabai, R., Olfson, M., Sampson, N. A., Jin, R., Druss, B., Wang, P. S., . . Kessler, R. C. (2011). Barriers to mental health treatment: Results from the National Comorbidity Survey Replication. Psychological Medicine, 41, 1751-1761.

Nyamathi, A. M. \& Flaskerud, J. (1992). A community-based inventory of current concerns of impoverished homeless and drug-addicted minority women. Research in Nursing and Health, 15, 121-129. 
Pennebaker, J. W. \& Graybeal, A. (2001). Patterns of natural language use: Disclosure, personality, and social integration. Current Directions in Psychological Science, 10, 9093.

Rappaport, J. (1995). Empowerment meets narrative: Listening to stories and creating settings. American Journal of Community Psychology, 23, 795-807.

Reeves, W. C., Strine, T. W., Pratt, L. A., Thompson, W., Ahluwalia, I., Dhingra, S. S., ... \& Safran, M. A. (2011). Mental illness surveillance among adults in the United States. MMWR Surveillance Summary, 60, 1-29.

Rosenberg, M. (1979). Conceiving the Self. New York: Basic Books.

Roth, A. M., Hensel, D. J., Fortenberry, J. D., Garfein, R. S., Gunn, J. K. L., \& Wiehe, S. E. (2014). Feasibility and acceptability of cell phone diaries to measure HIV risk behavior among female sex workers. AIDS and Behavior, 18, 2314-2324.

Roth, A. M., Rosenberger, J. G., Reece, M., \& Van Der Pol, B. (2013). Expanding sexually transmitted infection screening among women and men engaging in transactional sex: The feasibility of field-based self-collection. International Journal of STD and AIDS, 24, 323-328.

Schmitt, D. P. \& Allik, J. (2005). Simultaneous administration of the Rosenberg Self-Esteem Scale in 53 nations: Exploring the universal and culture-specific features of global selfesteem. Journal of Personality and Social Psychology, 89, 623-642.

Seal, A., Minichiello, V., \& Omodei, M. (1997). Young women's sexual risk taking behaviour: Re-visiting the influences of sexual self-efficacy and sexual self-esteem. International Journal of STD and AIDS, 8, 159-165.

Sobel, D. S. (1995). Rethinking medicine: Improving health outcomes with cost-effective psychosocial interventions. Psychosomatic Medicine, 57, 234-244.

StataCorp (2011). Stata Data Analysis Statistical Software: Release 12. StataCorp LP, College Station, TX.

Stopka, T. J., Springer, K. W., Khoshnood, K., Shaw, S., \& Singer, M. (2004). Writing about risk: Use of daily diaries in understanding drug-user risk behaviors. AIDS and Behavior, 8, 73-85.

Twenge, J. M. \& Campbell, W. K. (2001). Age and birth cohort differences in self-esteem: A cross-temporal meta-analysis. Personality and Social Psychology Review, 5, 321-344.

Ward, H., Day, S., \& Weber, J. (1999). Risky business: Health and safety in the sex industry over a 9 year period. Sexually Transmitted Infections, 75, 340-343.

Zimet, G. D., Dahlem, N. W., Zimet, S. G., \& Farley, G. K. (1988). The multidimensional scale of perceived social support. Journal of Personality Assessment, 52, 30-41. 